\title{
57. Specific Protein Combining with Yellow Pigments (Dihydropteridine) in the Silkworm, Bombyx mori L. ${ }^{1)}$
}

\author{
By Mitsuo Tsujita and Susumu SakuraI \\ (Comm. by Yoshimaro TANAKA, M.J.A., April 12, 1963)
}

In a previous study (in press), we have shown that the $+^{d-l e m}$ gene does not participate directly in the reaction of pteridine metabolism, but it seems to control the system that maintains the pteridine compound within the hypodermal cells. A hypodermis has been presented that the $+^{d-l e m}$ gene controls the production of a specific protein that is an important factor in combining with yellow pigments (2-amino-4-hydroxy-6-lactyl-7, 8-dihydropteridine) and in keeping them within the cells. To confirm this hypothesis the present experiments were conducted.

I. Materials and Methods. The test insects were four wild (normal) strains, C 124, N 124, Daizo and P $22\left(+^{l e m} /+^{l e m} ;+^{d-l e m} /\right.$ $\left.+^{d-l e m}\right)$, the lemon strain (lem/lem; $\left.+^{d-l e m} /+^{d-l e m}\right)$ and the dilute lemon strain (lem/lem; d-lem/d-lem).

(1) Preparation of chromogranules. A large amount of spherical granules, that contain yellow pigments and isoxanthopterin, occur in fresh hypodermal cells of larvae of the strains lemon and dilute lemon. The presence of these chromogranules in the hypodermal cells gives a yellow color to the larvae (Sasaki 1959, Tsujita 1961). Similarly, a large amount of spherical granules, that contain isoxanthopterin, can be observed in fresh hypodermal cells of larvae of the normal strain. These chromogranules were purified by a new method described in another paper, and lyophilized.

(2) Paper electrophoresis. A pellet $(10 \mathrm{mg})$ of the lyophilized sample of purified granules was mixed thoroughly under refrigeration with $1 \mathrm{ml}$ distilled water and then centrifuged at 15,000 r.p.m. for 40 minutes. A small amount $(0.05 \mathrm{ml})$ of the supernatant was painted on the line of application of Tōyo filter paper No. $51(26 \times 12.5 \mathrm{~cm})$ that was used for the paper electrophoresis. Electrophoresis was carried out for 5-6 hours at $\mathrm{pH} 8.6$ and 8.8 in Veronal buffer $(\mu=0.05)$ with $0.3 \mathrm{~mA} / 26 \mathrm{~cm}$. The protein bands were stained with bromophenol blue (B.P.B.). Prior to the staining of the protein bands, the localization of substances that showed fluorescence, such as isoxanthopterin and the yellow pigment, was observed by exposing the paper to ultraviolet light.

(3) High voltage electrophoresis. In order to detect the presence

1) Contribution from the National Institute of Genetics, Japan No. 474. 
of amino acids and peptides which contaminate the chromoprotein and to analyze the constitution of the protein of the chromogranules, the same samples used in the above electrophoresis experiment (2) were painted on the application line of Tôyo filter paper No. $51,60 \times 10 \mathrm{~cm}$ in size, which had been previously immersed in pyridine-glacial acetic acid-water mixture (volume ratio $1: 10: 90$ ) at $\mathrm{pH}$ 3.6. The treated paper was examined by high voltage electrophoresis under refrigeration in $\mathrm{n}$-hexane for 40 minutes. A $0.2 \%$ ninhydrin acetone solution was used for the staining of peptides and amino acids.

(4) Electrophoresis after the treatment of tryptic digestion. The purified sample was digested with trypsin (NBC salt free) at $\mathrm{pH}$ 8.0. The weight ratio of trypsin to protein was $1: 20$ or $1: 50$. The sample was dissolved in sodium-borate-boric acid buffer solution and heated for 3 minutes at $90^{\circ} \mathrm{C}$. After cooling, one to two drops of toluene and trypsin were added and the sample was incubated for 20 hours at $25^{\circ} \mathrm{C}$. The supernatant was examined by high voltage electrophoresis which had been described above.

II. Experimental results. 1. Chromogranules. The chromogranules obtained from either the cytoplasm of hypodermal cells of lemon larvae or those of the dilute lemon larvae were almost the same in shape, that is, they were irregularly spherical of $2-3 \mu$ in diameter (Fig. 1). The granules obtained from the hypodermis of larvae with the genotype, lem/lem; $+{ }^{d-\text { lem }} /+^{d-\text { lem }}$ had a much darker yellow color

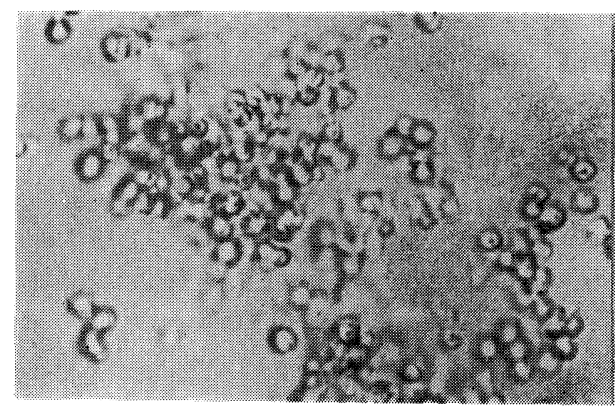

Fig. 1. Chromogranules purified from the hypodermal cells of lemon larvae with the genotype, lem/ lem; $+^{a-l e m} /+^{a-l e m} \times 1200$.

than those obtained from larvae with the genotype, lem/lem; d-lem/ $d$-lem. Aruga (1950) reported that uric acid occurred in the spherical granules and that the yellow pigments were found in minute clavateshaped particles. In the present study, however, all the purified spherical granules had a distinctly yellow color that indicated the presence of yellow pigments. Similarly shaped granules were obtained from the hypodermal cells of normal larvae by the above purification procedure. The diameter of these granules was 1-2.5 $\mu$, and was smaller than those of the granules obtained from the other two 
mutant larvae. The granules from normal larvae were white, and contained a large amount of isoxanthopterin.

2. Paper electrophoresis. Experimental results of paper electrophoresis are given in the following figure.

As shown in Fig. 2, the chromoprotein from larvae of the normal strain remained at the application line. A large portion of the substances that exhibited violet fluorescence were observed at the protein region, but a portion had advanced toward the anode. On the contrary, the chromoproteins from larvae of the lemon and the dilute lemon strains had advanced to the anode, but the movement of the dilute lemon chromoprotein was not as distinct as that of the lemon chromoprotein. Moreover, the yellow pigments remained close to the protein region and indicated an intimate relationship with the protein. This relationship is seen in Fig. 2.

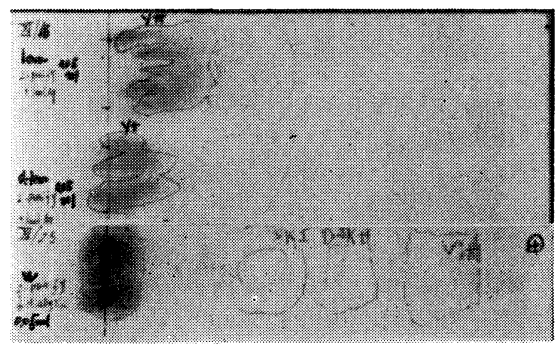

Fig. 2. Results of paper electrophoresis of chromoprotein prepared from the hypodermal celles of normal, lemon and dilute lemon larvae. w: normal (wild) strain. lem: lemon strain. d-lem: dilute lemon strain. sk, vi, $y$ mean the localization of substances that showed fluorescence.

3. High voltage paper electrophoresis. The protein band that had a positive reaction for B.T.B. was detected close to the application line but two or three spots that exhibited a positive reaction for ninhydrin had advanced toward the cathode. Substances that exhibited fluorescence were found in the protein band.

Since it was necessary to remove the peptides or amino acids which were present as contaminants, the samples were dialyzed with a visking dialysis membrane. After the low molecular weight substances were completely removed from the samples, the pure granular proteins were digested with trypsin, and then examined by high voltage paper electrophoresis. The experimental results are shown in Fig. $3 \mathrm{~A}$, B.

As shown in Fig. $3 \mathrm{~A}$, the peptide bands were not clearly observable on the upper surface of the filter paper, but their patterns could be separated one from the other on the lower surface of the paper. The patterns are diagrammatically shown in Fig. $3 \mathrm{~B}$. Seven bands each of which contained different peptides could be discriminated in the patterns of the protein from the wild (normal) strain. On the other hand, two supernumerary bands in addition to the common seven bands were present in the pattern of the protein from the 


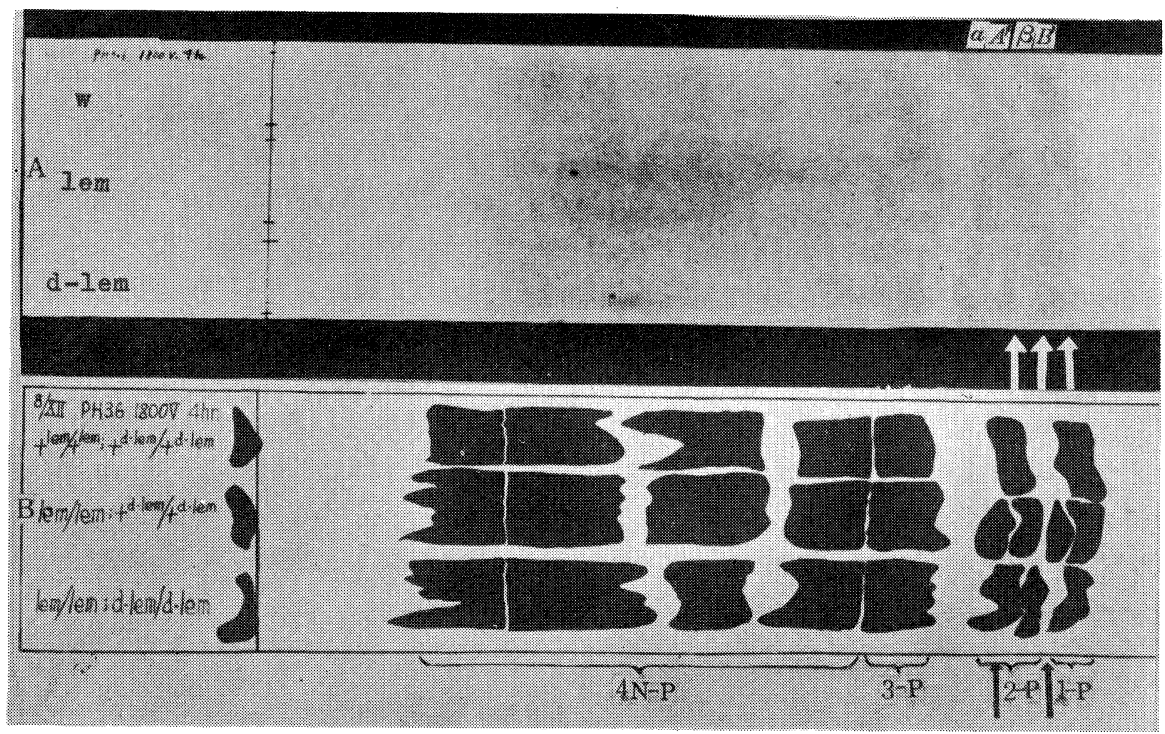

Fig. 3. A, B. Results of high voltage electrophoresis of the samples digested with trypsin.

A: photograph of upper surface of the filter paper.

$\mathrm{A}^{\prime}, \mathrm{B}^{\prime}$ : common peptide bands which occurred in the three strains.

$\alpha^{\prime}, \beta^{\prime}$ : specific peptide bands which occurred in the lemon or the dilute lemon strain.

B: diagrammatical figure of the lower surface of the filter paper.

w: normal strain. lem: lemon strain. $d$-lem: dilute lemon strain.

lemon strain. The protein from the dilute-lemon strain, however, exhibited only one supernumerary band.

The above patterns on the filter paper were divided into four parts, 1-P, 2-P, 3-P and 4N-P as shown in Fig. 3 B. To obtain a certain amount of peptides in each of 4 parts, concentrated samples were painted on the application line of the filter paper and analyzed by high voltage electrophoresis. Peptides present in each of the four parts were eluted by $1 \mathrm{M}$ acetic acid solution and condensed. The analysis of peptides in each fraction was carried out by the descending method of paper chromatography with the use of n-butanol-glacial acetic acid-water (volume ratio $4: 1: 5$ ) as solvent. A part of the experimental results are shown in Fig. $4 \mathrm{~A}, \mathrm{~B}$.

As seen in Fig. $4 \mathrm{~A}$, in 1-P of the lemon sample, one supernumerary peptide band appeared in addition to the 10 common ones. In the 2-P of the lemon and the dilute lemon samples, a supernumerary peptide band whose $\mathrm{Rf}$ value differed with the two samples appeared in addition to the 10 common ones (Fig. $4 \mathrm{~B}$ ). These specific peptides could not be observed in the normal sample. Thus, clearcut differences were found in the peptide composition of the three types of chromoprotein. 


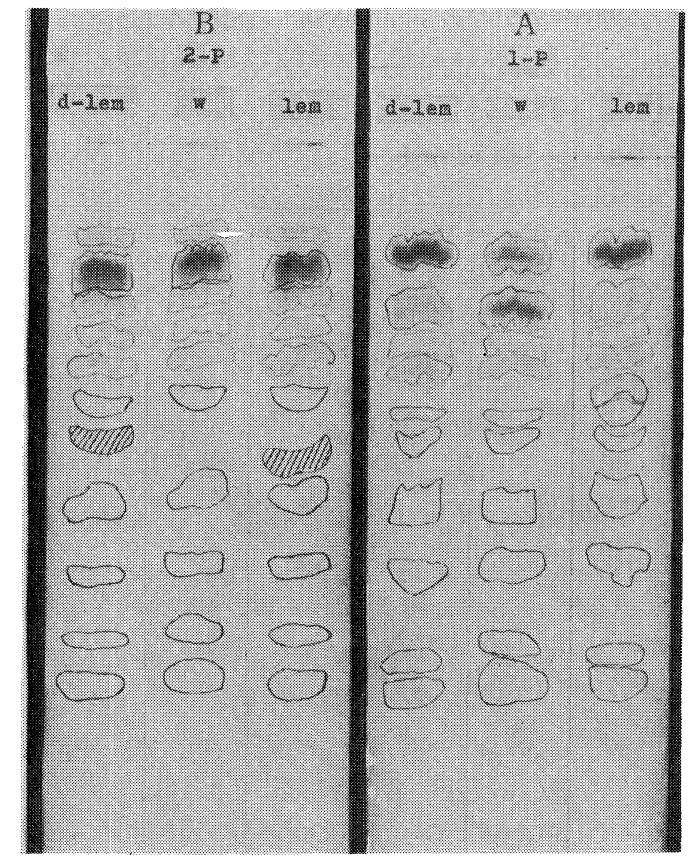

Fig. 4. The results of the peptide analysis of the two parts, 1-P and 2-P, shown in Fig. 3 by the descending method of paper chromatography.

Bands indicated by oblique lines mean supernumerary peptides which was specifically produced in the lemon and the dilute lemon larvae.

A. Peptide analysis of 1-P.

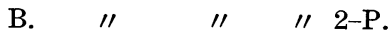

w: normal strain.

lem: lemon strain.

$d$-lem: dilute lemon strain.

III. Discussion. The yellow pigments whose structure was studied by Nawa (1961) are found within chromogranules which are formed abundantly in the cytoplasm of hypodermal cells of the lemon and the dilute lemon larvae. Furthermore, in the cytoplasm of hypodermal cells of normal larvae, there are similar spherical granules but they are smaller in size than those of the lemon and the dilute lemon larvae. These granules from normal larvae contain a large amount of isoxanthopterin. It is apparent that the chromogranules absorb and combine with the pteridine compounds, such as dihydropterin and isoxanthopterin.

The paper electrophoresis investigation of chromogranules of normal, lemon, and dilute lemon larvae showed that the granules from each type of larvae had a characteristic pattern. It was at first thought that these electrophoretic differences might reflect small structural dissimilarities among the three types of chromoprotein, comparable to those that have been found in mutant human haemoglobin (Ingram 1958). However, the digestion of the chromoprotein with trypsin showed differences in the peptide composition; the chromoprotein of the lemon sample contained two supernumerary peptides in addition to those commonly observed in the three types of chromoprotein, while the chromoprotein of the dilute lemon sample had only one additional peptide that had a different $\mathrm{Rf}$ value when compared with the supernumerary one produced by the $2-\mathrm{P}$ of the lemon sample. 
The two supernumerary peptides found in the lemon sample are believed to be components of the specific protein which has the ability to combine with yellow pigments. The single supernumerary peptide found in the dilute lemon sample is apparently a component of the specific protein which has the ability to combine with yellow pigments. The single supernumerary peptide found in the dilute lemon sample is apparently a component of the specific but an altered protein.

On the basis of the present study, the biochemical interactions between lem and $+^{d-l e m}$ or between lem and $d$-lem genes may be explained as follows. The yellow pigments are produced abundantly in hypodermal cells in which the step leading from dihydropterin to tetrahydropterin is blocked by the lem gene (Tsujita 1961). A specific protein is produced under the control of $+^{d-l e m}$ gene action in the presence of yellow pigment. As the chromogranules in hypodermal cells of larvae with the genotype lem/lem; $+{ }^{a-l e m} /+^{d-l e m}$ contain this protein, they are able to absorb yellow pigments and keep them within the cells. On the other hand, the altered protein produced under the control of $d$-lem gene action is present in the chromogranules of the dilute lemon larvae. Consequently in the dilute lemon larvae, the ability of the granules to absorb and retain the yellow pigments is markedly decreased, and such larvae have a dilute yellow color.

Summary. The chromoprotein composition of the three types of chromogranules obtained from normal, lemon and dilute lemon larvae has been examined by paper electrophoresis, and characteristic differences have been found in their electrophoretic mobility. Furthermore, characteristic differences have been found in the peptide composition of the three types of granules. In the lemon larvae a specific protein that seems to have the ability to combine with yellow pigments has been found, and in the dilute lemon larvae the specific but an altered protein has been found. However, this specific protein could not be observed in the normal larvae.

\section{References}

Aruga, H. (1950): Jap. J. Genet., 25(1-2), 76-77.

Forrest, H. S., and S. Nawa (1962): Nature, 196(4852), 372-373.

Ingram, V. M. (1958): Biochem. Biophys. Acta, 28, 539-545.

Nawa, S. (1960): Bull. Chem. Soc. Japan, 33, 1955-1960.

- (1960): Ann. Rep. Nat. Inst. Genet., 11, 32.

Sasaki, S. (1959): Bull. Seric. Exp. Sta., 15(5), 239-336.

Tsujita, M. (1961): Jap. J. Genet., 36(9-10), 337-346.

- Ibid. (in press).

—: and S. Sakurai: Ibid. (in press). 